\title{
Droplet Contact Angle Measurement on Microstructures of Octocoral Sclerites-ESEM Image Analysis
}

\author{
Barkay Zahava $^{1 *}$, Golombick Roy ${ }^{2}$, Yasmin Gabay $^{3}$, and Benayahu Yehuda ${ }^{3}$ \\ 1. Wolfson Applied Materials Research Center, Tel. Aviv. University, Ramat Aviv, Tel Aviv 69978, Israel \\ 2. Materials Engineering Nanotechnology program, Tel. Aviv. University, Ramat Aviv, Tel Aviv 69978, Israel \\ 3. Department of Zoology, George S. Wise Faculty of Life Sciences, Tel. Aviv. University, Ramat Aviv, Tel Aviv 69978, Israel
}

\begin{abstract}
This work explores the methodology for micron-scale water droplet contact angle derivation for the warty surface of octocoral sclerites. The calcite-made sclerites of the Red Sea octocoral Dendronephthya hemprichi have been chosen as a model for this study. Water droplet condensation on the sclerites has been in-situ investigated using Quanta 200 FEG (field emission gun) ESEM (environmental scanning electron microscope) under wet environmental conditions. Two different analysis methods of droplet top and side views have been applied to determine the contact angle based on the secondary electron images. The ESEM image analysis for the sclerites indicates that their surface is hydrophilic. The microscopic contact angle is measured to be $45.3^{\circ} \pm 6.3^{\circ}$. The macroscopic contact angle has been calculated by using the Wenzel model for the surface texturing of the sclerites.
\end{abstract}

Key words: Droplet, contact angle, octocorals, Dendronephthya hemprichi, ESEM.

\section{Introduction}

Corals are divided into two: hard corals (stony corals, or scleractinians), which are the major reef-builders featuring rigid calcium carbonate skeleton and octocorals (fleshy soft corals and sea fans), which have numerous tiny sclerites embedded in soft fleshy matrix. The importance of the reefs is as wave breakers, protection against floods, land-erosion and devastating storms such as hurricanes and typhoons. While alive, the reefs provide habitat for a high diversity of organisms of both invertebrates and fish. Coral reefs are also known for their economic importance, being source for food and touristic activities.

Global environmental changes due to fossil fuel burning and other sorts of pollution result with ocean acidification. This phenomenon is caused by emission of $\mathrm{CO}_{2}$, which in turn forms carbonic acid when dissolved in seawater and thus lowers the ocean $\mathrm{pH}$ levels. It thus threatens further calcification of reef-building corals and other calcifiers. Previous

\footnotetext{
* Corresponding author: Zahava Barkay, Ph.D., research field: electron microscopy.
}

work [1] indicated that fleshy tissues of octocorals may act as a barrier against skeleton corrosion under acidic conditions. This was tested against isolated sclerites, which underwent dissolution damage related to water acidity. The erosion damage of octocorals has a tight connection to their wettability properties [2].

The exoskeleton of stony corals which feature high content calcium carbonate scaffolds was shown to be biocompatible, osteo-conductive and biodegradable, which also depends on the porosity, implantation site and the specific coral species. In particular, corals were studied as potential bone graft substitutes in animals in the early 1970s and afterwards in humans in 1979 [3]. The biocompatibility of the calcium carbonate coral skeleton in respect to cell adhesion requires characterization of coral structures on the relevant cell size and in comparable to their surface inhomogeneity scale, i.e., at the micron-scale [4].

In this work, the spindle-shaped sclerites of the Red Sea octocoral Dendronephthya hemprichi [2] were studied by measuring the wettability properties on micron-scale. These calcite-made sclerites, which feature wary surface-microstructures, have been 
chosen for this study [5]. The droplet condensation on the sclerites has been in-situ imaged in the ESEM (environmental scanning electron microscope) under wet environmental conditions in similarity to the research of Aronov, et at. [6]. At this characteristic scale, the typical drop size is below the capillary length. The effect of gravity is thus neglected and the droplet acquires a perfectly spherical cap shape.

Two different analysis methods for top and side views $[7,8]$ were applied to determine the microscopic contact angle based on the secondary electron ESEM images. The methods were compared to provide a methodology for micron-scale contact angle derivation on Dendronephthya sclerites as depicted at that annual meeting [9]. Macroscopic contact angle measurements were previously reported on flat calcium carbonate surfaces by using calcium and carbonate ions in supersaturated solutions for preparation of calcium carbonate coatings [10]. In the current study, the macroscopic contact angle was calculated by the Wenzel model for rough surfaces [11], using the measured microscopic angle and the surface structure of the sclerites. The calculated macroscopic contact angle value was compared with optical goniometer measurements.

\section{Experiments}

\subsection{Experimental Set Up}

Samples of Dendronephthya hemprichi were collected from the coral reef across from the Interuniversity Institute for Marine Sciences in Eilat (IUI), Gulf of Aqaba, northern Red Sea. The sclerites were obtained by dissolving the tissues in $10 \%$ sodium hypochlorite, followed by careful rinsing in distilled water. The isolated sclerites were dried out and mounted on the ESEM cooling stage.

The current research was focused on in-situ micron-scale imaging of calcite-made sclerites. Imaging was performed in the Quanta 200 FEG ESEM using the GSED (gaseous secondary electron detector) with Peltier cooling stage $[9,12]$. Sample imaging was done at $2^{\circ}$ and 5.3 torr while in-situ condensation was performed at an elevated pressure up to 5.8 torr.

\subsection{Micro-contact Angle Analysis Methods}

Two ESEM image analysis methods have been used to measure the contact angle. The first analysis method corresponds to top view measurement (view parallel to primary electron beam axis). The secondary electron intensity profile along the droplet diameter is based on the model of Stelmashenko, et al. [7] and fitted to Eq. (1):

$$
I(\mathrm{x})=I_{0}\left(1-\frac{\left(X-X_{\mathrm{C}}\right)^{2}}{a^{2}} \sin ^{2} w\right)^{-1 / 2}+I_{\mathrm{B}}
$$

where, the $w$ is contact angle, $a$ is the apparent radius of the droplet base, $x_{\mathrm{c}}$ is the position of its center, $I(x)$ is the pixel intensity measured from an electron micrograph, $I_{0}$ and $I_{\mathrm{B}}$ are the intensity coefficients related to the normal intensity of secondary electron emission and background levels, respectively. The droplet base radii a and the position of droplet center $x_{\mathrm{c}}$ can be read off the image scan for each droplet, when the intensity at the droplet center was taken as $I_{0}+I_{\mathrm{B}}$. The intensity value at the ESEM image across the center of each droplet allows extracting the value of the contact angle $w$, which is the only fitting parameter in Eq. (1). Similar approach was performed for in-situ droplet condensation on flat silicon samples before and after surface e-beam treatment for hydrophilic and hydrophobic-like contact angle derivation [6].

The surface of Dendronephthya hemprichi sclerites is not flat and can rarely be tilted to produce an exact top view or side view images. Droplets formed on the warty surface are at an arbitrary angle relative to the incident beam. An alternative mathematical model thus provides extracting the real contact angle from SE images for spherical cap drops observed from an arbitrary angle. This model correlates the real contact angle $w$, the measured angle $\delta$, and the substrate inclination angles $z$ versus horizontal direction [8]:

$$
w=2 \tan ^{-1}\left[\frac{\sqrt{(\tan (\delta / 2))^{2}+(\cos z)^{2}-1}+\tan (\delta / 2)}{\cos z+1}\right]
$$




\section{Results and Discussions}

\subsection{ESEM-Characteristic Morphology}

A portion of a typical spindle-shaped sclerite of Dendronephthya hemprichi is presented in Fig. 1. It can be seen that the protruding features of the sclerite are roughly $25 \mu \mathrm{m}$ apart, $10 \mu \mathrm{m}$ in height and $10 \mu \mathrm{m}$ in width as schematically presented at Fig. 2.

\subsection{ESEM-Wettability Top View Measurement}

Upon in-situ condensation, droplets appear on the sclerites (Fig. 3a). Some drops were tilted with respect to the top view, while others were usually asymmetrical due to an interaction with a protruding feature or

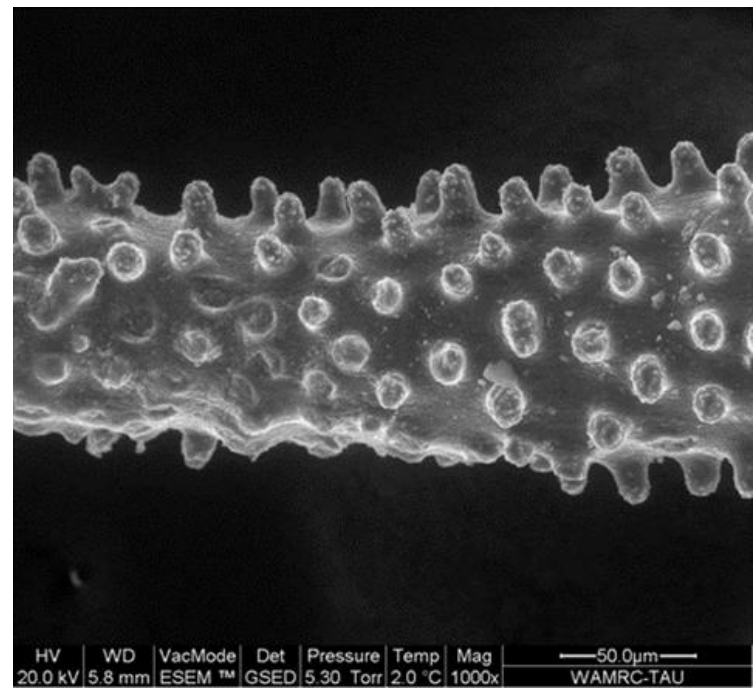

Fig. 1 Spindle shaped sclerite of Dendronephthya hemprichi prior droplet condensation.

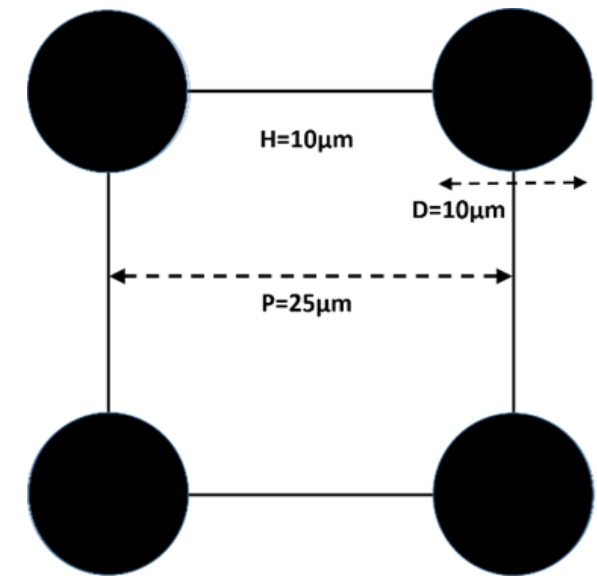

Fig. 2 Schematic basic unit structure of sclerite of the octocoral Dendronephthya hemprichi.
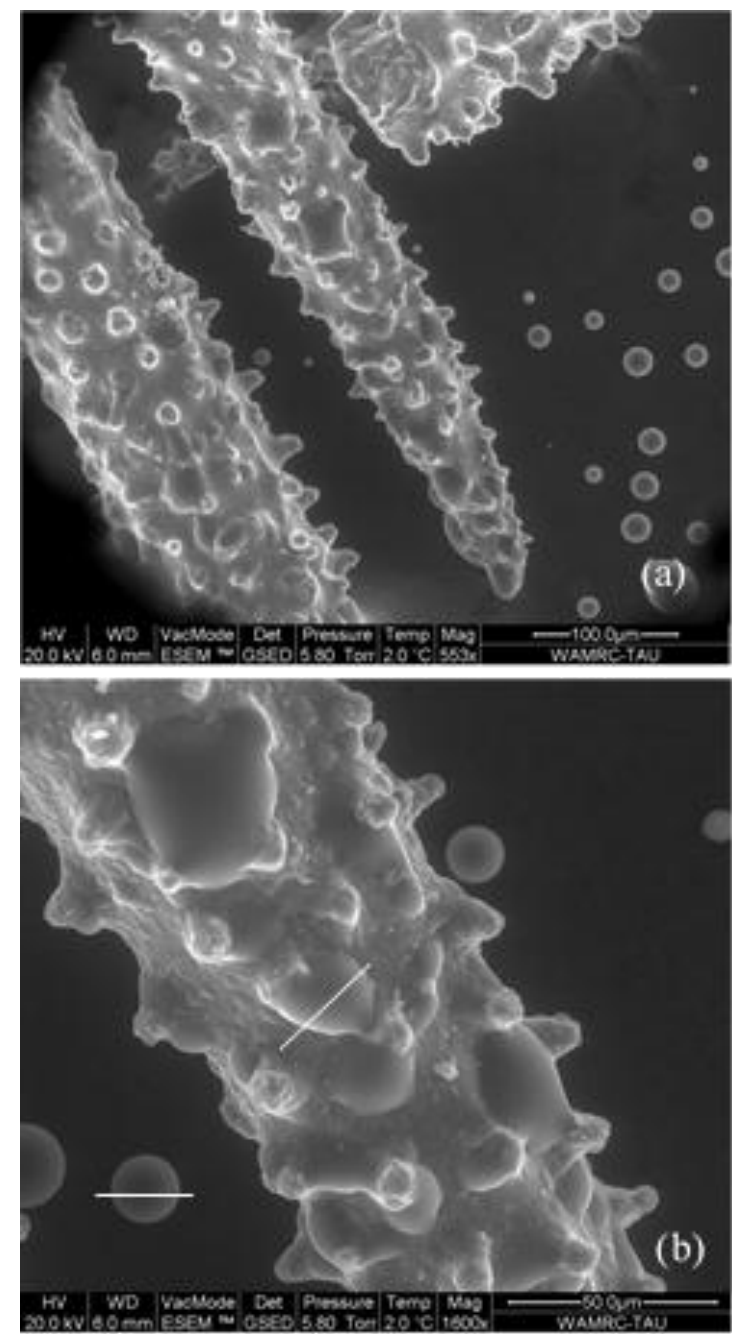

Fig. 3 In-situ condensation on sclerite of Dendronephthya hemprichi: (a) drops on the sclerite are misshaped in contrast to droplet on the carbon (on the right); (b) a magnified image of the middle part of the sclerite.

apparent defects on the surface. In comparison, the droplets formed on the carbon flat substrate itself are highly symmetrical and can be analyzed according to Stelmashenko's top view model [7] for contact angle (Fig. 3a). Fig. 4 shows a typical comparison of intensity profile for the droplet on the sclerite surface relative to a reference drop on the carbon substrate (corresponding to two lines at Fig. 3b). The characteristic comparison is a function of position with fit to Eq. (1) for the contact angle derivation. The reference drop on carbon flat surface (Fig. 4a) provided a typical contact angle of $57^{\circ}$ carbon, while the specific drop on the sclerite was characterized by asymmetric 

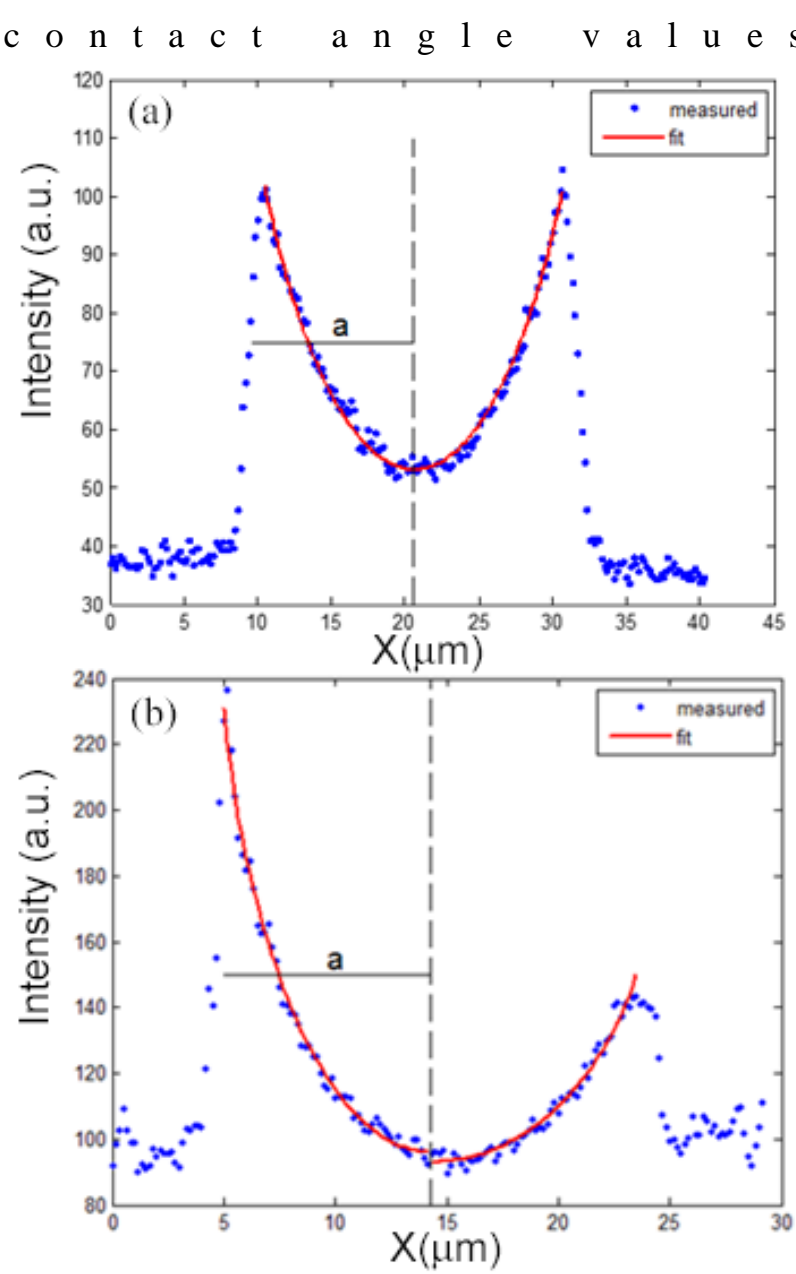

Fig. 4 Gray level intensity (arbitrary units) along the two lines chosen in Fig. 3b: (a) for drop on carbon; (b) for drop on the sclerite of Dendronephthya hemprichi.

(of $74^{\circ}$ and $65^{\circ}$ ) for both left and right sides of the profile (Fig. 4b). Additional measurements on the sclerites resulted in wide spread values, which required an alternative evaluation method.

\subsection{ESEM-Wettability Side View Measurement}

Side view contact angle measurements on octocoral Dendronephthya hemprichi sclerites structures are shown in Fig. 5. The real contact angle is extracted by using Brugnara's method for the measured and the substrate inclination angles according to Eq. (2). The results of overall 22 droplets provided a Gaussian distribution with contact angle of $45^{\circ} \pm 6^{\circ}$. The chosen micro-droplets were from regions in-between the protruding features, thus presenting the Young contact

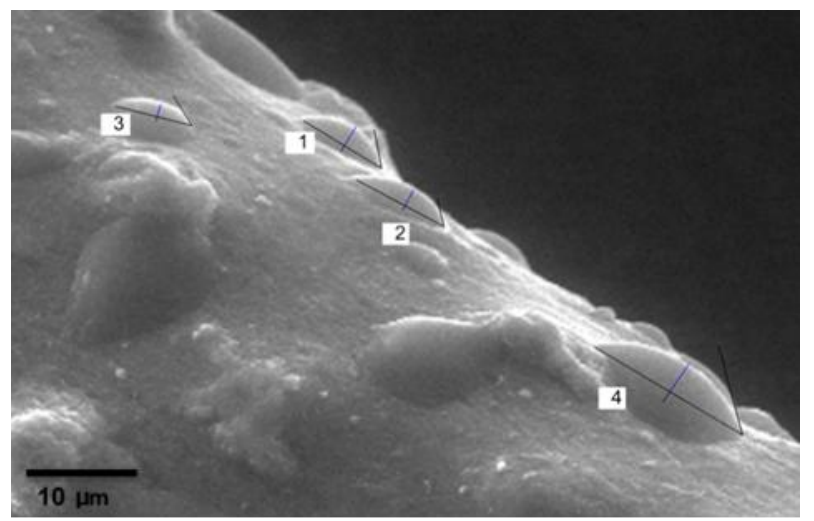

Fig. 5 Side view of four droplets on sclerite of Dendronephthya hemprichi showing the measured contact angles.

angle for a smooth surface structure. This micron-scale measurement thus corresponds to the intrinsic material properties. Using these results, predictions for macroscopic wetting properties, which depend on morphology, could be attained.

\subsection{Calculation of Macroscopic Contact angle Based on Wenzel Model}

The structure was modeled based on the ESEM images and the corresponding schematic basic unit of Dendronephthya hemprichi sclerites (Fig. 2). For simplicity, the structure was modeled assuming a two dimensional periodic structure of protruding feature height $\mathrm{H}=10 \mu \mathrm{m}$, diameter $\mathrm{D}=10 \mu \mathrm{m}$ and separation $\mathrm{P}=25 \mu \mathrm{m}$. The Young contact angle of $w \sim 45^{\circ}$ was used for a smooth surface. The apparent macroscopic contact angle $w_{\mathrm{m}}$ for the modeled structure was related to the Young angle $w$ by including the roughness effects [13]:

$$
\cos w_{\mathrm{m}}=\left(1+\frac{\pi D H}{P^{2}}\right) \cos w
$$

Substituting: $\mathrm{w} \sim 45^{\circ}, \mathrm{D}=10 \mu \mathrm{m}, \mathrm{H}=10 \mu \mathrm{m}, \mathrm{P}=25 \mu \mathrm{m}$, provided complete wetting $w_{m} \sim 0$ under current assumptions and in correlation to optical goniometer measurements. It is thus indicated that the surface roughness of the octocoral Dendronephthya hemprichi improves the wetting properties towards complete wetting. 


\section{Conclusions}

The ESEM provides a high spatial resolution and a relatively large depth of field of tens microns, which has been required for characterization of the rough surface of Dendronephthya hemprichi sclerites before and after in-situ droplet condensation.

The methodological research for micro-scale droplet measurement on the sclerites was based on comparing the top view and the side view ESEM measurements. The top view method resulted in a wide spread of contact angles due to drop coverage of the inhomogeneous surface. The droplets chosen for analysis in the side view method could far outnumber the droplets which can be analyzed in the top view method. Thus, unlike flat substrates, the in-situ side view droplet contact measurement in ESEM was found recommended over top view measurement for the rough surface of the Dendronephthya hemprichi sclerites. The statistics of the microscopic contact angle was of Gaussian distribution with mean value of $45.3^{\circ}$ and a standard deviation error of $6.3^{\circ}$. This value was within the range of prior work on macroscopic contact angle of calcium carbonate flat surfaces [10].

The chosen micro-droplets at side view measurements were in-between the protruding features of the sclerites, providing the intrinsic wetting properties of the calcite-made sclerites. The micron-scale contact angle of about $45^{\circ}$ differs from the macroscopic zero contact angle by optical goniometer. The difference is explained by including the surface roughness using the Wenzel model for apparent contact angle. The results thus show that the sclerite surface roughness of octocoral Dendronephthya hemprichi induces complete wetting.

Under non-active sea conditions, the skeleton of the sclerites is completely embedded in the soft fleshy matrix as could be explained by complete wetting, especially for the highly viscous fleshy matrix. Further analysis would include the dependence of the sclerite wetting properties on the sea water $\mathrm{pH}$ levels and on the skeleton surface treatments. The microstructures of octocoral sclerites is highly variable [4], which could affect their surface roughness, wettability properties and potential usage as biocompatible materials.

\section{Acknowledgments}

The authors thank the Wolfson Applied Materials Center and the center for Nano Science and Nanotechnology for feasibility study in the environmental scanning electron microscope. The study was in part supported by The Israel Cohen Chair in Environmental Zoology to Y.B. The authors thank the Interuniversity Institute for Marine Sciences in Eilat (IUI) for facilities. Collection of animals complied with a permit issued by the Israel Nature and Parks Authority.

\section{References}

[1] Gabay, Y., Fine, M., Barkay, Z., and Benayahu, Y. 2014. "Octocoral Tissue Provides Protection from Declining Oceanic pH.” Plos. One 9 (4): 1-7.

[2] Fabricius, K. E., and Alderslade, P. 2001. "Soft Corals and Sea Fans: a Comprehensive Guide to the Tropical Shallow-Water Genera of the Central West Pacific, the Indian Ocean and the Red Sea." Townsville, Australia: Australian Institute of Marine Science.

[3] Demers, C., Hamdy, C. R., Corsi, K., Chellat, F., Tabrizian, M., and Yahia, L. 2002. "Natural Coral Exoskeleton as Bone Graft Substitute: A Review." Bio-Medical Materials and Engineering 12: 15-35.

[4] Tentori, E., and Van. Ofwegen, L. P. 2011. "Patterns of Distribution of Calcite Crystals in Soft Coral Sclerites." Journal of Morphology 272: 614-28.

[5] Grossowicz, M., and Benayahu, Y. 2011. "Differential Morphological Features of Two Dendronephthya Soft Coral Species Suggest Differences in Feeding Niches." Marine Biodiversity 42 (1): 65-72.

[6] Aronov, D., Rosenman, G., and Barkay, Z. 2007. "Wettability Study of Modified Silicon Dioxide Surface using Environmental Scanning Electron Microscopy." Journal of Applied Physics 101: 084901-5.

[7] Stelmashenko, N. A., Craven, J. P., Donald, A. M., Terentjev, E. M., and Thiel, B. L. 2001. "Topographic Contrast of Partially Wetting Water Droplets in Environmental Scanning Electron Microscopy." Journal of Microscopy 204 (2): 172-83.

[8] Brugnara, M., Volpe, C. D., Siboni, S., and Zeni, D. 2006. "Contact Angle Analysis on Polymethylmethacrylate and 
Commercial Wax by using an Environmental Scanning Electron Microscope." Scanning 28 (5): 267-73.

[9] Golombick, R., Gabay, Y., Benayahu, Y., and Barkay, Z. 2013. "Droplet Contact Angle Measurement on Dendronephthya Structures-ESEM Image Analysis." In Proceedings of the 47th Annual Meeting of the Israel Society of Microscopy.

[10] Orkoula, M. G., Koutsoukos, P. G., Robin, M., Vizika, O., and Cuiec, L. 1999. "Wettability of $\mathrm{CaCO}_{3}$ Surfaces." Colloids and Surfaces A: Physicochem. Eng. Aspects 157: 333-40.
[11] De Gennes, P. G., Brochard-Wyart, F., and Quere, D. 2003. Capillarity and Wetting Phenomena. Germany: Springer.

[12] Donald, A. M. 2003. "The Use of Environmental Scanning Electron Microscopy for Imaging Wet and Insulating Materials." Nature Materials 2: 511-6.

[13] Jung, Y. C., and Bhushan, B. 2008. "Wetting Behavior during Evaporation and Condensation of Water Microdroplets on Superhydrophobic Pattered Surfaces." Journal of Microscopy 229 (1): 127-40. 